\title{
Post Colectomy Ileitis in patients with Ulcerative \\ Colitis: A Diagnostic Challenge
}

\author{
Antakia $\mathbf{R}^{1 *}$, Toquero $\mathrm{L}^{1}$, Lishman $\mathrm{S}^{2}$ and Makhija $\mathbf{R}^{1}$ \\ ${ }^{1}$ Department of General \& Colorectal Surgery, Peterborough and Stamford Hospitals NHS Foundation \\ Trust, Peterborough, UK \\ ${ }^{2}$ Department of Histopathology, Peterborough and Stamford Hospitals NHS Foundation Trust, \\ Peterborough, UK
}

Received: 10 April, 2020

Accepted: 20 April, 2020

Published: 21 April, 2020

*Corresponding author: Mr Ramez Antakia, Division of General Surgery, Health Education England, East of England Deanery, MBChB, MRCS Ed, MSc, MPhil,

9 Martin Reed Walk, Bury St Edmunds, Suffolk, IP32 6FG, United Kingdom, Tel: (+44) 07983336264;

E-mail: antakia26@hotmail.co.uk

https://www.peertechz.com

\section{Check for updates}

\begin{abstract}
Aims: Post-colectomy ileitis is a common yet challenging disease entity. We present our management approach in these complex patients.

Methods: We present a case series of four patients with post-colectomy ileitis in patients with Ulcerative Colitis (UC).

Results: Despite optimal medical therapy, these patients underwent subtotal colectomy with end ileostomy and were discharged with tapering steroid regimen. They represented acutely (2-4 weeks) or chronically (months to years) with severe abdominal pain and/or problematic stoma output. Three patients required re-laparotomies, with two requiring further small bowel resection. Histology revealed necrosis and perforation but no granulomas. Diagnosis of post-colectomy ileitis was made. Both patients who underwent further bowel resection responded well to steroid therapy with Total Parenteral Nutrition (TPN) and remain on endoscopic surveillance. Symptomatic control was achieved with systematic/local steroid therapy in the remaining two patients.
\end{abstract}

Discussion/Conclusion: Steroid therapy with TPN is the mainstay of treatment in these patients to address nutritional needs especially those requiring multiple laparotomies.

Post-colectomy ileitis should be considered as a differential in UC patients post total colectomy with persistent abdominal symptoms and exclusion of other types of ileitis should be examined with histological and radiological investigations. Multi-disciplinary approach is mandatory and management of these patients in randomised clinical/controlled trial (RCT) setting is required.

\section{What does this paper add to the existing literature}

We present a case series of four patients with post-colectomy ileitis in patients with ulcerative colitis to highlight the existence of this condition. Despite optimum medical therapy, patients who underwent subtotal colectomy with end ileostomy, represented with severe abdominal symptoms. We adopted multidisciplinary approach including patient's preference and close follow-up in managing this challenging complex condition.

\section{Introduction}

Post-operative ileitis following total colectomy is an uncommon yet serious condition. This represents a diagnostic challenge and often the original pathology of the inflammatory bowel disease should be revisited. Initial cases of postcolectomy ileitis were reported over 50 years ago. Since then it has been described to exist in both acute (two to four weeks following surgery) and chronic (months to years post surgery) forms although only few reports exist. Reported incidence varies, with some authors feeling that it is under-recognised [1-3]. Unfortunately the reported re-operative rates are high and the mortality associated with the condition can be as high as $25 \%$ [1]. The current case series highlights the presence of this disease entity and the difficulty in management despite a combined medical and surgical approach.

We have four patients in our case series with age ranging 
from 26 to 55 years, each with a background history of UC. Histological diagnosis was established at least one year prior to a diagnosis of post-colectomy ileitis. Despite best medical management, and following a joint gastroenterology and colorectal multidisciplinary team (MDT) discussion, a decision was made to proceed with laparoscopic or open subtotal colectomy and formation of an end ileostomy. Each patient represented back to the emergency department acutely or chronically with severe abdominal pain and increased stoma output with or without bleeding. Three patients required re-laparotomies, with two requiring further small bowel resection. Histology revealed multiple patches of necrosis and perforation, but no evidence of granulomas or skip lesions. In each case, a diagnosis of post-colectomy ileitis was made.

Our first case is that of a 26 year-old female, previously on Mesalazine suppositories who presented with fulminant UC, and despite initial treatment with intravenous Hydrocortisone proceeded to a laparoscopic subtotal colectomy and end ileostomy. The Terminal Ileum (TI) was noted to be macroscopically normal, whilst histology confirmed UC, with a normal TI. She recovered well and was discharged 8 days after surgery with a reducing steroid regimen. Two weeks post-discharge, the patient presented again as an emergency with severe abdominal pain and passing copious amounts of liquid stool mixed with blood per stoma. Urgent Computed Tomography (CT) scan showed oedematous terminal ileum at the ileostomy with fat stranding and a localised collection. She underwent another emergency laparotomy where ischaemic patches were noted within $25 \mathrm{~cm}$ of the ileostomy requiring further resection of small bowel. Histology showed evidence of haemorrhagic necrosis and acute inflammatory cells infiltration with no evidence of granulomas or dysplasia or malignancy. The patient responded well to further steroid therapy and Total Parenteral Nutrition (TPN).

Our second case is that of a 47 year-old male with UC who presented similarly acutely with severe abdominal pain and increasing stool frequency. He was treated with intravenous Hydrocortisone but despite this, he continued to deteriorate clinically. He subsequently underwent to a subtotal colectomy with end ileostomy, histology confirming UC. The patient was discharged 15 days after surgery on a reducing regimen of steroids. The patient presented four weeks later to the emergency department with severe abdominal pain and raised inflammatory markers. The patient represented a second time with worsening abdominal pain, high output stoma and faeculant discharge from the side of the stoma. He was taken to theatre for a re-laparotomy, and perforation was found at the side of the terminal ileum, which was also thickened and dusky in appearance with multiple mucosal ulcerations. Histology showed mild chronic active inflammation with cryptitis. The ileostomy was re-fashioned and the patient was discharged nine days following his second laparotomy managing his stoma normally.

Our third case is a 45 year-old male who was initially diagnosed with pancolitis in 2007 and underwent panproctocolectomy and an end ileostomy in 2008. Histology showed active chronic inflammation with foci of cryptitis and superficial ulceration with no granulomas, dysplasia or malignancy in favour of UC. He re-presented within four weeks with abdominal pain, nausea and intermittent watery stoma output which settled down with medical treatment. In 2011, the patient opted to have an ileo-anal pouch and he recovered relatively well following this surgery. He continued to suffer from intermittent abdominal pain and diarrhoea, which was suspected to represent flare ups of his original Inflammatory Bowel Disease (IBD) or terminal ileitis for which he still uses both systemic and topical steroid therapy and undergoes routine 6-12 monthly endoscopic surveillance.

Our fourth case is a 55 year-old female, also initially diagnosed with non-specific pancolitis in May 2014. Despite best medical therapy, she continued to remain symptomatic and underwent a laparoscopic subtotal colectomy with end ileostomy within ten days of her acute admission. Histology showed active chronic inflammation most severe in the distal colon, foci of cryptitis, haemorrhagic changes with surface ulceration and depletion of submucosal fat, but no granulomas or dysplasia or malignancy, which is consistent with UC. The patient recovered well from her surgery and was discharged home with a reducing steroid regimen. She was re-admitted within four weeks of her initial surgery with worsening abdominal pain and worsening watery stoma output up to eight times daily. Urgent ileoscopy revealed evidence of ileitis and the patient responded well to steroid therapy. The patient continued to complain with rectal pain and mucous bloody discharge per rectum requiring an expedited laparotomy and completion proctectomy. Histology showed active inflammation with areas of superficial ulceration with no granulomas, dysplasia or malignancy.

\section{Investigations}

In all patients, detailed history taking and stool samples were sent in order to exclude other differentials such as drug reaction, infective colitis or Crohn's disease. Initial histology in all four patients showed evidence suggestive of UC. On representation, CT with IV contrast of the abdomen and pelvis was performed to look for possible intra-abdominal collections in the post-operative period. Ileoscopy was performed via the stoma and ileal mucosa demonstrated severe erythema and ulceration extending $25-30 \mathrm{~cm}$ from the stoma (Figures 1-3). Histology revealed active chronic inflammation, foci of cryptitis with areas of haemorrhage and superficial ulceration. In the first two patients who underwent re-laparotomy, the decision to re-operate was indicated by clinical presentation such as rigid abdomen, faeculant discharge from around the stoma together with urgent CT findings as in the first case. Intraoperative findings in those two patients showed and oedematous terminal ileum with multiple patches of ischaemic necrosis and areas of microperforation respectively. Histology of the resected TI of each case brought back to theatre demonstrated areas of severe non-specific ileitis with no evidence of granuloma formation, as well as focal ulceration and inflammation extending into the muscularis propria. The fourth patient underwent expedited completion proctectomy due to persistent active symptomatic disease in 
the rectal stump. Patients underwent surveillance ileoscopy three weeks following discharge which showed no evidence of any significant lesion, but did demonstrate non-specific active chronic ileitis. Both the third and fourth patients had magnetic resonance enterography and surveillance endoscopy in the consequent years, which showed good clinical remission of the original UC. However the last MRI for the third patient in 2018 showed mild narrowing at $20 \mathrm{~cm}$ proximal to the pouch anastomosis, but the patient remains in clinical remission.

\section{Differential diagnosis}

The diagnosis of post-colectomy ileitis was made based on the clinical presentation of these patients along with the

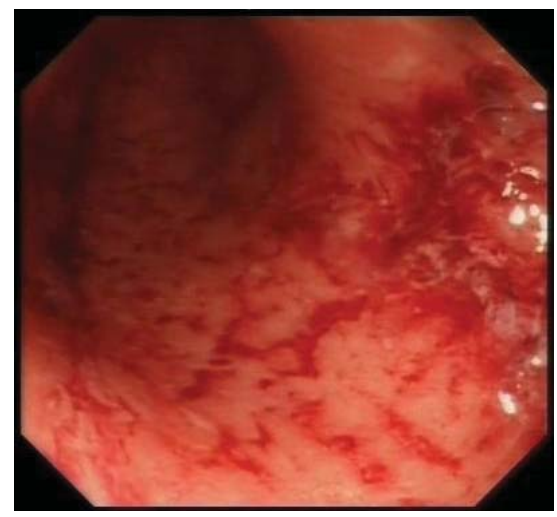

Figure 1: Terminal ileitis with linear haemorrhages.

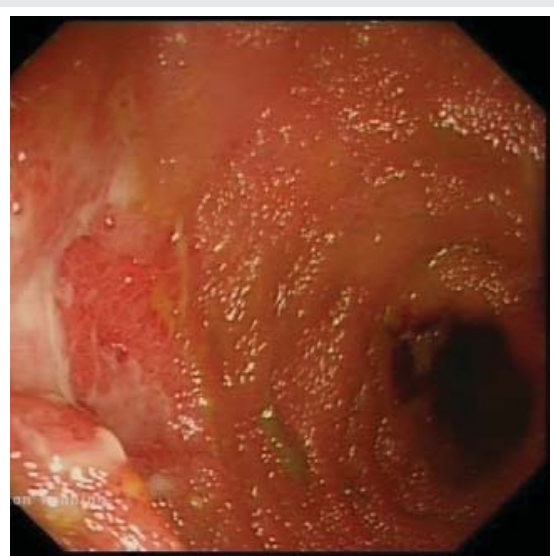

Figure 2: Severe terminal ileitis with mucosal ulcers and shedding

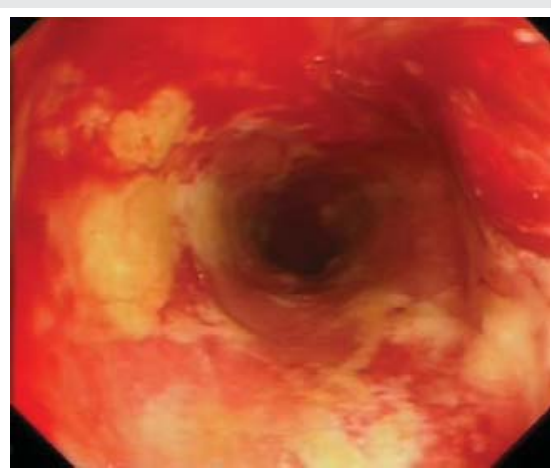

Figure 3: Terminal ileitis with superficial haemorrhage and patchy ulceration. intraoperative findings at laparotomy and the histological findings of the resected small bowel. The differential of Crohn's disease (CD) was entertained, but histopathological features did not support this. Furthermore none of the patients in our small cohort developed small bowel strictures or fistulae in our five-year follow-up period.

\section{Management}

The diagnostic challenge under these circumstances of post-operative re-admissions was whether the original diagnosis of UC was correct or whether CD could have been a possibility. Ileoscopy in each case revealed extensive areas of deep ulceration in the terminal ileum. Following a short postop stay, each patient subsequently received enteric feeding, after an initial short course of TPN and was subsequently discharged symptom-free. All four patients are still undergoing endoscopic surveillance to ensure optimum treatment and detect early signs of disease flare up.

\section{Discussion}

There are few reported incidences of post-colectomy ileitis over the last 60 years [1-8]. The condition occurs following colectomy for UC, and can present either acutely within two to four weeks or chronically up to several years following the index procedure.

One group from The Cleveland Clinic has provided the bulk of published work on this subject $[1,2]$ although the disease was first described by Thayer and Spiro in 1962 [3]. Over a number of papers, they have attempted to refine the understanding of this post-surgical syndrome of UC. Aetiology behind post-colectomy ileitis is still unclear and various explanations have been suggested in the literature such as infection, vasculitis, ischaemia, drug-related, backwash ileitis (unlikely post-operative) or multi-factorial [10]. Some authors have postulated that the incidence of post-colectomy ileitis may be related to the extent of the disease prior to surgery [1].

The disease presents in one of two ways, either as an acute or chronic condition $[1,4]$. The chronic condition tends to occur over months to years of normal ileostomy function following the initial surgery usually presenting with diarrhoea, weight loss and stoma dysfunction. Stoma-related symptoms are secondary to chronic ulceration and subsequent fibrosis of the terminal ileum, stoma retraction, fistula formation, chronic obstruction and dilatation [1].

Acute presentation has been reported within 10 to 14 days of the initial operation $[1,9]$. Fever, tachycardia and watery, often blood-stained stoma output were the predominant symptoms observed within this subgroup of patients. The authors reported that perforation occurred in $50 \%$ of the patients within this group and that these perforations were multiple and found on the anti-mesenteric border [1]. They have also reported that the extent of the disease process was difficult to determine because in their experience the external surface of the ileum "appeared relatively uninvolved".

Parallels can clearly be drawn between the signs and 
symptoms as well as the operative features of the acute form of post-colectomy ileitis described by Hawk, et al. (1970). The patients presented with both clinical and histopathological signs found by other authors. However, despite the reported high mortality rates for patients presenting with perforation, we found that the use of TPN and high dose steroids in the post-operative period helped improve the patients' morbidity by meeting their nutritional needs while allowing the bowel to recover.

The clinical dilemma with patients with postcolectomy ileitis is the prevention of future occurrences and the level of follow-up. Hawk, et al. (1970), have postulated that intramuscular administration of 40 units of adrenocorticotrophin gel post-operatively once daily for the first week followed by twice weekly thereafter has apparently eliminated the presentation of the acute form of the disease in their experience by dampening the immunologic response implicated in the transmural and mucosal colitis [1]. The efficacy of this hormone in management of post-colectomy ileitis needs to be studied in a Randomised Controlled Trial (RCT) with adequate long term follow up. One study has shown that a number of treatments used routinely for suppression of inflammatory bowel disease (including 5-aminosalicylic acid and oral steroids) were ineffective in treating this condition [9], whilst other studies reported that Azathioprine was effective as it provided both symptomatic and endoscopic improvement $[9,11]$. However these findings have only been reported in relatively small case series and also would be better assessed in a RCT setting.

Difficulties in delivering effective treatment may stem from a lack of clarity regarding the underlying pathological process which drives post-colectomy ileitis. In many cases, the ileitis is a recurrence of the disease process for which the initial operation was done $[1,9]$. In others, the histology revealed a non-specific inflammatory process [1], but this doesn't mean it is not the same original disease. A recent study with at least a 10 -year follow-up period suggested that in up to $25 \%$ of patients undergoing colectomy for UC initially, the disease diagnosis was revised to be $\mathrm{CD}[12]$.

Post-colectomy ileitis is a commonly encountered condition that remains challenging in surgical practice. Its acute presentation can be fatal to the patient if not acted upon quickly. Better understanding of this condition would make it possible to diagnose sooner and hence commence earlier management and decrease associated morbidity. The paucity of available literature on the subject demands the need for management strategies to be formally investigated in the future in randomised trials.

\section{Take home messages}

- Post-colectomy ileitis should be considered a differential in UC patients post total colectomy who present with persistent abdominal symptoms.

- Although little is known about the underlying pathology, limited small bowel resection with TPN appears to be the mainstay of treatment.

- Reconsideration of the diagnosis of Crohn's disease should be undertaken with histological and radiological investigations.

- A multi-disciplinary approach in making the diagnosis and subsequent management is mandatory.

\section{References}

1. Hawk A, Turnbull R, Schofield P (1970) The Natural History of Post-Colectomy Ileitis. Proc R Soc Med 63: 68-73. Link: https://bit.ly/2XKwGDg

2. Turnbull R, Weakley F, Farmer R (1964) Ileitis after Colectomy and Ileostomy for Nonspecific Ulcerative Colitis: Report of 35 Cases. Dis Colon Rectum 7: 427-35. Link: https://bit.ly/2yrXOre

3. Thayer WR, Spiro HM (1962) lleitis after ileostomy: prestomal ileitis. Gastroenterology 42: 547-55. Link: https://bit.ly/2wRusqw

4. Rogers AG, Bargen JA, Black BM (1954) Chronic ulcerative colitis: early and late experiences of 124 patients with ileal stomas. Gastroenterology 27: 383 398. Link: https://bit.ly/2VlyqB3

5. Lyons AS, Garlock HJ (1954) The complications of ileostomy. Surgery 36: 784789.

6. Scott AD, Phillips RKS (1989) lleitis and pouchitis after colectomy for ulcerative colitis. Br J Surg 76: 688-689. Link: https://bit.ly/2VjPqrs

7. Colcock BP, Mathiesen WL (1956) Complications of surgical treatment of chronic ulcerative colitis. Arch Surg 72: 399-404. Link: https://bit.ly/3eDpej4

8. Tolstedt GE, Bell JW (1961) Intestinal obstruction following total colectomy for ulcerative colitis. Am Surg 153: 241-245. Link: https://bit.ly/2wRThTg

9. Hallak A, Baratz M, Santo M, Halpern Z, Rabau M, et al. (1994) lleitis after Colectomy for Ulcerative Colitis or Carcinoma. Gut 35: 373-376. Link: https://bit.ly/3alQRtN

10. DiLauro S, Crum-Cianflone NF (2010) lleitis: when it is not Crohn's disease. Curr Gastroenterol Rep 12: 249-258. Link: https://bit.ly/2VkmzDA

11. Hoentjen F, Hanauer SB, Hart J, Rubin DT (2013) Long-term treatment of patients with a history of ulcerative colitis who develop pan-enteritis after colectomy. J Clin Gastroenterol 47: 52-57. Link: https://bit.ly/3amJ5jp

12. Shamah S, Schneider J, Korelitz BI (2018) High incidence of recurrent crohn's disease following colectomy for ulcerative colitis revealed with long follow-up. Dig Dis Sci 63: 446-451. Link: https://bit.ly/2yvaQsE 